\title{
Versatility of the Distally-Based Sural Artery Fasciocutaneous Flap on the Lower Leg and Foot in Patients with Chronic Disease
}

\author{
Jin-Su Park, Si-Gyun Roh, Nae-Ho Lee, Kyoung-Moo Yang \\ Department of Plastic and Reconstructive Surgery, Chonbuk National University School of Medicine, Jeonju, Korea
}

Background A recent advancement in microsurgery, the free flap is widely used in the reconstruction of the lower leg and foot. The simple and effective methods of local flaps, including transposition and advancement flaps, have been considered for patients with chronic debilitation who are unable to endure long surgical procedures or general anesthesia. However, the location and size of the wound may restrict the clinical application of a local flap. Under these circumstances, a sural flap can be an excellent alternative, rendering satisfying clinical outcomes in chronically debilitated patients.

Methods Between 2008 and 2012, 39 patients underwent soft tissue defect treatment by sural artery flap as a final method. All of the patients had at least one chronic disease or more (diabetes, hypertension, vascular disease, etc.). Also, all of the patients had a history of chronic lower extremity ulceration, which revealed no response to several months of conservative treatment.

Results The results of the 39 cases had a success rate of 100\% with 39 complete recoveries. Nine cases suffered complications: partial necrosis $(n=4)$, wound dehiscence without necrosis $(n=3)$, hematoma $(n=1)$, and infection $(n=1)$.

Conclusions The sural artery flap is not only useful for the lower leg but also for the heel, and other various parts. Furthermore, it is a relatively simple surgical technique for reconstructing the defect area for patients with various chronic conditions with a high surgical risk or contraindications to surgery.

Keywords Sural / Soft tissue injuries / Surgical flap

\author{
Correspondence: Si-Gyun Roh \\ Department of Plastic and \\ Reconstructive Surgery, Chonbuk \\ National University School of \\ Medicine, 20 Geonji-ro, Deokjin-gu, \\ Jeonju 561-712, Korea \\ Tel: +82-63-250-1860 \\ Fax: +82-63-250-1866 \\ E-mail: pssroh@jbnu.ac.kr
}

This article was presented at the 69th Congress of the Korean Society of Plastic and Reconstructive Surgeons on November 9-11, 2012 in Seoul, Korea.

No potential conflict of interest relevant to this article was reported.

\section{INTRODUCTION}

Coverage of soft tissue defects of the lower leg and foot remains a challenging yet common reconstructive problem [1]. The frequency of the procedure has increased with the increasing incidence of chronic diseases (diabetes, hypertension, vascular disease, etc.) that affect this location. There are various options for covering soft tissue defects. Therapeutic options include the local, regional, and free flaps, with each method having drawbacks that limit its clinical application. The use of local flaps, including transposition and advancement flaps with random vascularity, is restricted because of the limited amount of tissue movable from areas adjacent to the defect and limited flap mobilization [2].

Thus reconstruction by a free flap transfer is superior to other 
methods, as it can provide well vascularised tissue of any size with minimal donor site morbidity. However, it has the disadvantage of being a sophisticated and lengthy procedure, involving a high price, advanced equipment, a microsurgical team, and the application to chronic disease patients, for small to moderate defects $[3,4]$.

For these reasons, we have considered other options including musculocutaneous flaps and muscle flaps; the distally based superficial sural artery fasciocutaneous flap has been identified as a good alternative. The use of a fasciocutaneous flap from the sural angiosome in the repair of soft tissue defects was first reported by Ponten [5] in his 1981 report on the use of 23 proximally based fasicocutaneous flaps. The distally based sural fasciocutaneous flap was introduced by Donski and Fogdestam [6] 2 years later. In 1987, Taylor and Palmer [7] introduced the angiosome concept. Furthermore, the sural angiosome, as one of the four angiosomes of the leg, was introduced by Taylor and Pan [8] about 11 years later. The skin, the bones, and most muscles received branches from two or more angiosomes, thus revealing one of the important anastomotic pathways through which the circulation is reconstituted when a source artery is interrupted by disease or trauma [8]. This anatomical study contributes further information to help design or redesign flaps in the lower leg. Since then, a variety of anatomical studies have contributed to our knowledge of the procedure.

Since 2008, we have performed 39 distally based superficial sural artery fasciocutaneous flaps. The recipient sites have included the lower leg, ankle, heel, lateral malleolus, medial malleolus, and foot. We here present our experience of the distally based superficial sural artery fasciocutaneous flap.

\section{METHODS}

\section{Patients and method}

There is no simple rule for when a defect can and cannot be covered. The basic consideration in the surgical decision making is the defect size and pedicle length. This study was performed with the following criteria: 1) general condition of patients (include the medical condition and extent of control of chronic disease), 2) preoperative evaluation (laboratory findings, image studies, etc.), 3) compliance of patients to surgery and a course of treatment, 4) level of difficulty of using general anesthesia for surgery.

The occlusion of the anterior tibial artery, posterior tibial artery, and peroneal artery should be considered contraindications of surgery.

Preoperative management for a successful outcome was performed by X-ray, 3-phase bone scan, and Doppler sonography.
We also performed computed tomography angiography when abnormal findings such as vascular stenosis and an occlusive pattern in Doppler sonography were found. In addition, we measured the ankle brachial index and performed percutaneous transluminal angiography in the cardiovascular risk group (over 60, diabetes mellitus, hypertension, and chronic kidney disease, and average of ankle-brachial index $[\mathrm{ABI}]$ value: $0.4-0.8$ [normal range, 0.9-1.2]). In the case of those who were undergoing hemodialysis, we prevented an electrical imbalance by preoperative hemodialysis.

For the past 5 years, from 2008 to 2012, we treated 39 patients with skin and soft tissue defects. The treatment area included the lower leg, heel, ankle, lateral malleolus, medial malleolus, and foot. Of the 39 patients, 25 had diabetic ulcer (all patients had type II diabetes mellitus controlled by medication and insulin therapy), 21 had hypertension (all patients had essential hypertension controlled by medication), 10 had peripheral artery disease (complication of diabetes mellitus, hypertension, and the aging process), 7 had osteomyelitis (most patients have chronic osteomyelitis due to diabetic ulcers and it is almost impossible to eradicate bacterial infection), 5 had chronic kidney disease (all patients needed hemodialysis due to the kidney no longer working), and 1 had thyroid disease (hypothyroidism).

For each patient, we recorded the age, sex, etiology, medical conditions, size of lesion, and the operation results and any complications. The average age of the patients was 60 (ranging from 27 to 85). All of the patients either had an incompletely healed chronic ulcer despite several months of conservative treatments or an incurable ulcer in their medical history. Among the conservative treatments were regular wet dressing, negative pressure therapy, and use of casting and other materials. More aggressive treatments included excision of infected and necrotic tissues, medical debridement, and biological dressing. Sural flap was performed on patients whose deletion area was treated with a conservative method for closure, but which was not successful for several months (Table 1).

\section{Surgical technique}

The surigical technique has been described in detail in previous publications, and we describe it briefly here. The sural artery fasciocutaneous flap is made of skin and subcutaneous fat, superficial and deep fascia of the posterior part of the leg, sural nerve, saphenous vein, and the superficial sural artery [9].

First, the patient is situated in a prone position. Before inflating the pneumatic tourniquet, a sterile handheld Doppler is used to spot the peroneal artery perforators at the lateral malleolus. After marking the axis of the flap, which is the course of the medial sural nerve, the flap is outlined according to the size of the defect 
Table 1. The data of the 39 patients

\begin{tabular}{|c|c|c|c|c|c|c|c|c|}
\hline No. & $\begin{array}{l}\text { Age/ } \\
\text { Sex }\end{array}$ & $\begin{array}{l}\text { Defect } \\
\text { etiology }\end{array}$ & Defect site & $\begin{array}{c}\text { Medical } \\
\text { comorbities }\end{array}$ & $\begin{array}{l}\text { Wound defect } \\
(\mathrm{cm})\end{array}$ & $\begin{array}{l}\text { Donor site } \\
\text { closure }\end{array}$ & Wound complication & Outcome \\
\hline 1 & $65 / F$ & DU & Heel & $\mathrm{DM}$ & $5 \times 5$ & STSG & None & Good \\
\hline 2 & $54 / \mathrm{M}$ & Burn & Heel & DM, HTN & $6 \times 4$ & STSG & None & Good \\
\hline 3 & $64 / M$ & DU & Heel & DM, HTN, CKD & $5 \times 4$ & STSG & None & Good \\
\hline 4 & $69 / F$ & Burn & $\mathrm{LL}$ & HTN, Breast cancer & $10 \times 5$ & STSG & Hematoma & Good \\
\hline 5 & $55 / M$ & DU & LM & $\mathrm{DM}, \mathrm{OM}$ & $6 \times 4$ & STSG & None & Good \\
\hline 6 & $43 / M$ & Trauma & Heel & DM, Thyroid disease & $4 \times 5$ & Primary & None & Good \\
\hline 7 & $76 / M$ & PS & Heel & DM & $6 \times 7$ & STSG & None & Good \\
\hline 8 & $78 / \mathrm{M}$ & Burn & $\mathrm{LL}$ & HTN, PAD & $9 \times 7$ & STSG & None & Good \\
\hline 9 & $61 / M$ & Trauma & Heel & HTN & $5 \times 6$ & STSG & None & Good \\
\hline 10 & $36 / M$ & Trauma & Heel & DM, Hepatitis & $5 \times 4$ & STSG & None & Good \\
\hline 11 & $44 / M$ & DU & Heel & DM, PAD & $6 \times 4$ & STSG & Partial necrosis & Good \\
\hline 12 & $44 / M$ & Trauma & Foot & PAD & $5 \times 4$ & STSG & None & Good \\
\hline 13 & 79/M & DU & LM & DM, PAD & $5 \times 5$ & STSG & Wound dehiscence & Good \\
\hline 14 & $61 / M$ & Melanoma & $\mathrm{LL}$ & DM, HTN & $8 \times 5$ & STSG & None & Good \\
\hline 15 & $72 / \mathrm{F}$ & PS & Heel & DM, HTN, CKD & $5 \times 7$ & STSG & Partial necrosis & Good \\
\hline 16 & $27 / M$ & Trauma & LM & PAD & $5 \times 4$ & STSG & None & Good \\
\hline 17 & $85 / M$ & Melanoma & Ankle & DM, PAD & $4 \times 6$ & Primary & Infection & Good \\
\hline 18 & $39 / \mathrm{M}$ & PS & LM & Quadriplegia, HTN & $3 \times 3$ & Primary & None & Good \\
\hline 19 & $50 / M$ & DU & Ankle & DM, PAD & $4 \times 4$ & Primary & None & Good \\
\hline 20 & $33 / M$ & $\mathrm{OM}$ & MM & $\mathrm{OM}$ & $3 \times 2$ & Primary & None & Good \\
\hline 21 & $65 / F$ & DU & LM & DM, HTN & $4 \times 6$ & STSG & Partial necrosis & Good \\
\hline 22 & $40 / F$ & DU & LM & $\mathrm{DM}, \mathrm{CKD}$ & $4 \times 3$ & Primary & Infection & Good \\
\hline 23 & $71 / \mathrm{M}$ & Burn & MM & DM, HTN, PAD & $5 \times 2$ & STSG & None & Good \\
\hline 24 & $49 / F$ & DU & MM & $\mathrm{DM}, \mathrm{OM}$ & $2 \times 3$ & Primary & None & Good \\
\hline 25 & $62 / M$ & Trauma & Ankle & HTN & $4 \times 4$ & Primary & None & Good \\
\hline 26 & $55 / F$ & DU & LM & DM, HTN & $3 \times 3$ & Primary & None & Good \\
\hline 27 & $29 / F$ & Trauma & $\mathrm{LL}$ & $\mathrm{OM}$ & $5 \times 5$ & STSG & None & Good \\
\hline 28 & $58 / \mathrm{M}$ & Burn & Heel & DM, HTN, PAD & $5 \times 5$ & STSG & None & Good \\
\hline 29 & $76 / F$ & DU & Foot & $\mathrm{DM}, \mathrm{OM}$ & $4 \times 4$ & STSG & None & Good \\
\hline 30 & $73 / \mathrm{M}$ & Trauma & MM & HTN & $3 \times 3$ & Primary & None & Good \\
\hline 31 & $64 / M$ & Trauma & Heel & HTN, OM & $3 \times 5$ & STSG & None & Good \\
\hline 32 & $67 / F$ & Trauma & $\mathrm{LL}$ & HTN & $4 \times 8$ & STSG & Wound dehiscence & Good \\
\hline 33 & $49 / M$ & Burn & Ankle & $\mathrm{DM}$ & $5 \times 4$ & STSG & None & Good \\
\hline 34 & $68 / M$ & DU & Heel & DM, HTN & $4 \times 5$ & STSG & Partial necrosis & Good \\
\hline 35 & $63 / M$ & DU & LM & DM, HTN, CKD & $3 \times 4$ & Primary & None & Good \\
\hline 36 & $62 / M$ & Trauma & $\mathrm{LL}$ & HTN, OM & $7 \times 8$ & STSG & Wound dehiscence & Good \\
\hline 37 & $60 / F$ & Burn & MM & DM, CKD & $3 \times 3$ & Primary & None & Good \\
\hline 38 & $59 / F$ & Trauma & Foot & HTN, PAD & $4 \times 3$ & STSG & None & Good \\
\hline 39 & $75 / M$ & DU & Heel & DM, HTN & $5 \times 5$ & STSG & None & Good \\
\hline
\end{tabular}

and distance from it, along the posterior lower two thirds of the leg. The flap design is a teardrop shape rather than a matchstick shape to reduce compression of the pedicle (Fig. 1). Debridement of the recipient area is performed. The pivot point of the flap is about $5 \mathrm{~cm}$ above the lateral malleolus, proximal to the most distal perforators from the peroneal artery. The incision is made along the superior border of the flap. At mid-calf, the sural nerve and lesser saphenous vein are identified suprafascially. The sural nerve, artery, and lesser saphenous vein are divided and ligated, and included within the flap. The entire flap is elevated and dissected from the proximal to distal end. Dissection of the graft has to reach the distal limit as marked, $5 \mathrm{~cm}$ proximal to the lateral malleolus. The width of the carrier pedicle is 3 to 4 $\mathrm{cm}$. There is direct dissection from the donor site to the recipient site, rather than a subcutaneous tunnel (Fig. 2). The flap is then transposed to the area of the defect to be covered by carefully rotating the flap over its pedicle up to $180^{\circ}$. The pneumatic tourniquet is deflated, and the circulation in the flap is checked. The flap is then transposed distally and sutured to the receptor site. Depending on the flap condition, primary or delayed closure is used at the pedicle site. The donor area is either primarily closed or skin grafted, depending on the dimensions (Fig. 3). 


\section{Fig. 1. Teardrop-shaped design}

The flap is outlined according to the size and distance of the defect. The pivot point of the flap is approximately $5 \mathrm{~cm}$ above the lateral malleolus.

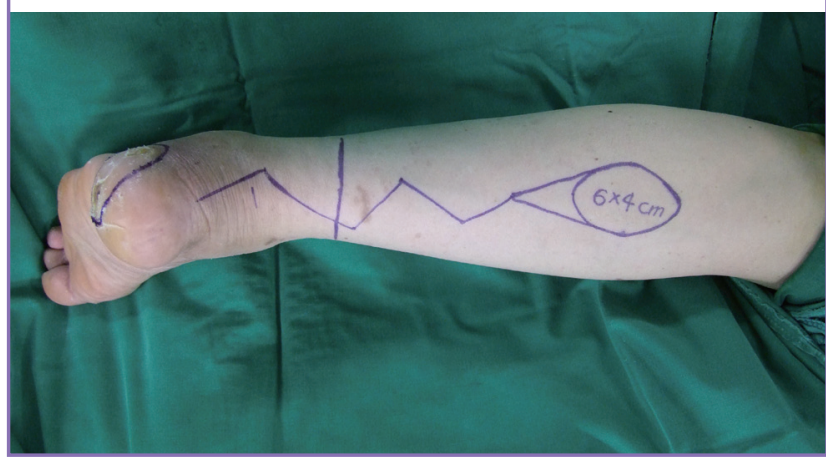

\section{Fig. 2. Flap dissection and elevation}

After debridement of the recipient area, the entire flap is elevated and dissected proximally to distally. The width of the carrier pedicle is 3 to $4 \mathrm{~cm}$.

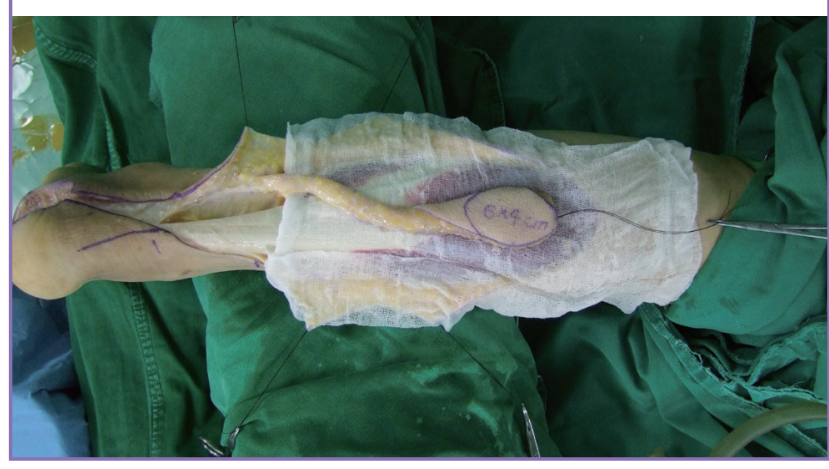

The protective splint with light but well-padded dressing is applied, ensuring that there is no compressionof the pedicle.

\section{RESULTS}

For the past 5 years, from 2008 to 2012, 39 cases of sural flap were performed on 39 patients. Among them, 27 patients were male, and 12 were female. The causes of the defect were diabetic ulcer for 14 patients, trauma for 12 patients, burn for 7 patients, pressure sore for 3 patients, melanoma for 2 patients, and osteomyelitis for 1 patient. The affected areas of the 39 patients were as follows: 13 patients were affected in the heel; 8 patients in the lateral malleolus; 6 patients in the lower leg; 4 patients in the ankle; 5 patients in the medial malleolus; and 3 patients in the foot. In all cases, the defect areas were covered with sural flap. The size of the flap for the operation was 2 to $10 \mathrm{~cm}$ in length and 2 to $8 \mathrm{~cm}$ in width.

The results of the sural artery fasciocutaneous flap for the 39 cases had a success rate of $100 \%$ with 39 complete recoveries.

\section{Fig. 3. Flap rotation and inset}

The flap is transposed to the defect area by carefully rotating the flap over its pedicle up to $180^{\circ}$. Then the recipient site is closed, and the donor site is either primarily closed or skin grafted.

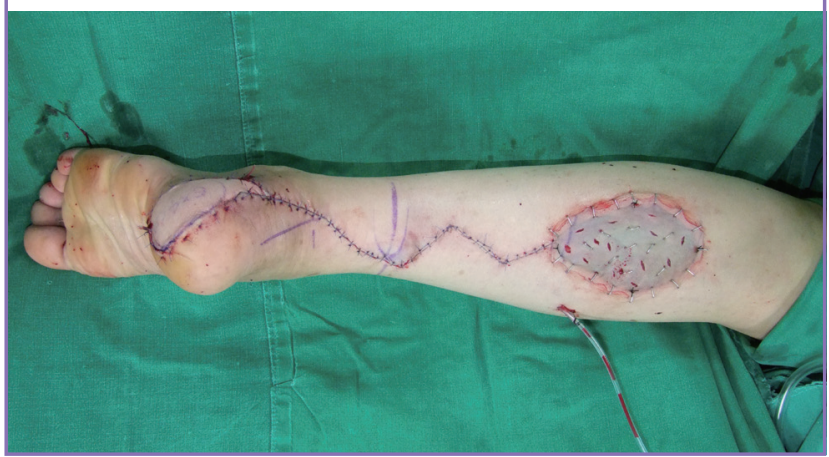

Fig. 4. Long term follow-up

Postoperative view of a patient after 10 months.

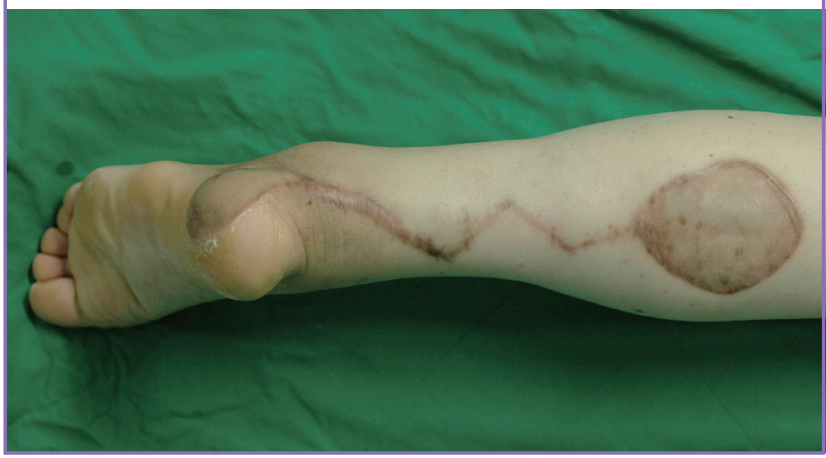

Complete recovery was defined as complete coverage of the defect area and primary closure of the donor area or maintenance of proper wound closure through skin grafting. A total of 9 patients suffered complications: 4 patients with partial necrosis; 3 patients with wound dehiscence without necrosis; 1 patient with hematoma; and 1 patient with an infection. For the 4 patients with partial necrosis, debridement or secondary closure was first attempted, thus healing 2 patients. The other 2 patients who were not healed with the first attempt had a skin graft to cover the necrotic tissue. There was one case of infection among the 39 flaps, but it was cured with a clinically appropriate antibiotic treatment based on a culture.

The total recovery period was approximately 2 to 3 weeks in those without any complications, while the cases with complications took an additional 2 to 3 weeks to recover (Fig. 4).

\section{DISCUSSION}

The reconstruction of the unresolved lower leg, heel, ankle, lateral malleolus, medial malleolus, and foot is a rather challenging task for reconstructive surgeons. It is not simply a matter of the 
affected area, as the chronic underlying disease affects the operation or the results of the operation, making the reconstruction difficult.

Generally, for an ideal flap, elevation and anastomosis of the flap should be easy and precise without damaging other structures such as the major arteries and nerves. It should also be possible to conduct without microsurgical technology.

Causes of a defect requiring surgery include diabetic ulcer, trauma, burn, melanoma, pressure sore, and osteomyelitis, and such lesions may be serious enough to expose ligaments, bone, blood vessels, and major structures. Such lesions cannot be covered with a skin graft, and most cannot be cured with surgical techniques such as a local flap, because there is a limit to flap mobilization or the presence of inappropriate tissues around the affected areas. For these reasons, a free flap is the first to consider when reconstructing a chronic defect area.

However, the sural artery fasciocutaneous flap is a useful and excellent alternative to a free flap, based on its advantages and applications. The sural artery fasciocutaneous flap can be an easier approach to reconstruction of various parts of the lower extremities. The reason is that during sural flap, an appropriate amount of blood is supplied without the use of the main vessels. In addition, patients with chronic conditions incompatible with general anesthesia can undergo an effective reconstruction operation with regional anesthesia for serious defects, such as exposure of the blood vessels, bone, and defective soft tissues. Moreover, a sural flap can be performed by one experienced surgeon and anatomically distant lesions such as the ankle or foot can be easily operated on with a long pedicle. Another great advantage is that a single operation can reconstruct the defect area without the technical aspects of microsurgery or the recently developed supermicrosurgery.

The disadvantage of sural flap was hypo- or hyperesthesia such as paresthesia or numbness caused by damage to the sural nerve. This problem appeared to gradually improve among the younger patients during the postoperative observation period. Another limitation was the aesthetic problem of scarring or cicatrix of the operated or donor area. It appeared to be more problematic in cases of a female patient requiring a skin graft, and if the first closure was not possible due to the size of the donor area.

As a general principle also applicable to other flaps, the absolute contraindication of sural artery fasciocutaneous flap is the apparent infection of the lesion. Osteomyelitis or uncontrollable chronic underlying disease of the patient is considered to be a relative contraindication. However, this study has displayed strong results with its 100\% full recovery rate, by regarding the relative contraindications that can cause complications after the operation as surgical indicators. Limited improvement in the patient's chronic condition or the stability of the lesion itself should be considered for a high rate of full recovery.

Although sural flap is an excellent method for reconstruction of defects of the lower extremities of patients with underlying chronic disease, there are inevitable complications. In this study, the occurrence of complications was significant, at $23 \%$. A similar analysis was conducted by Baumeister, et al. [10] of complications was $36 \%$. Complications included partial necrosis, wound dehiscence, hematoma, and infection, and this high rate of occurrence is considered to have a close relationship with the morbidity state of chronic disease. A number of studies have suggested that venous congestion and the lack of arterial supply are the most significant reasons for flap necrosis [10]. Tan et al. [11] recently described their technique in "supercharging" the sural flap. Simply, this technique is performed by anastomosing the lesser saphenous vein to any recipient area vein. Also, Wong and Tan [12] reported that intermittent phlebotomy was a simple and effective way to relieve venous congestion during the immediate postoperative period. However, in our study, the clamping of the proximal end of the lesser saphenous vein in preparation for supercharging was optional when the flap circulation was not good. Furthermore, the inclusion of the sural nerve and the short saphenous vein along the entire length of the flap is crucial to maximize the reliability of this flap [13]. Additional factors in maintaining vascularity and reducing complications are the teardrop-shaped design, the direct dissection from the donor site to the recipient site, rather than a subcutaneous tunnel, and the choice of pedicle site closure (primary, delayed, or skin graft).

Postoperative management is considered to be the most important. Careful attention to patient position in the early postoperative period, leg elevation, and pressure-free dressing can generate the ideal outcome.

Because it has been found that the number of patients with chronic disease is currently increasing, the distally based sural artery fasciocutaneous flap is a good alternative to free tissue transfer to consider for reconstruction of the lower leg and foot.

\section{REFERENCES}

1. Fraccalvieri M, Verna G, Dolcet M, et al. The distally based superficial sural flap: our experience in reconstructing the lower leg and foot. Ann Plast Surg 2000;45:132-9.

2. Chen SL, Chen TM, Wang HJ. The distally based sural fasciomusculocutaneous flap for foot reconstruction. J Plast Reconstr Aesthet Surg 2006;59:846-55.

3. Serafin D, Georgiade NG, Smith DH. Comparison of free flaps with pedicled flaps for coverage of defects of the leg or foot. Plast Reconstr Surg 1977;59:492-9. 
4. Swartz WM, Mears DC. The role of free-tissue transfers in lower-extremity reconstruction. Plast Reconstr Surg 1985; 76:364-73.

5. Ponten B. The fasciocutaneous flap: its use in soft tissue defects of the lower leg. Br J Plast Surg 1981;34:215-20.

6. Donski PK, Fogdestam I. Distally based fasciocutaneous flap from the sural region: a preliminary report. Scand J Plast Reconstr Surg 1983;17:191-6.

7. Taylor GI, Palmer JH. The vascular territories (angiosomes) of the body: experimental study and clinical applications. $\mathrm{Br}$ J Plast Surg 1987;40:113-41.

8. Taylor GI, Pan WR. Angiosomes of the leg: anatomic study and clinical implications. Plast Reconstr Surg 1998;102:599616.

9. Rios-Luna A, Villanueva-Martinez M, Fahandezh-Saddi H, et al. Versatility of the sural fasciocutaneous flap in coverage defects of the lower limb. Injury 2007;38:824-31.

10. Baumeister SP, Spierer R, Erdmann D, et al. A realistic complication analysis of 70 sural artery flaps in a multimorbid patient group. Plast Reconstr Surg 2003;112:129-40.

11. Tan O, Atik B, Bekerecioglu M. Supercharged reverse-flow sural flap: a new modification increasing the reliability of the flap. Microsurgery 2005;25:36-43.

12. Wong $\mathrm{CH}$, Tan BK. Intermittent short saphenous vein phlebotomy: an effective technique of relieving venous congestion in the distally based sural artery flap. Ann Plast Surg 2007;58:303-7.

13. Nakajima H, Imanishi N, Fukuzumi S, et al. Accompanying arteries of the lesser saphenous vein and sural nerve: anatomic study and its clinical applications. Plast Reconstr Surg 1999;103:104-20. 